\title{
Management of Penetrating Trauma to the Major Abdominal Vessels
}

\author{
Peep Talving ${ }^{1,2,3} \cdot$ Sten Saar ${ }^{1,2} \cdot$ Lydia Lam $^{4}$
}

Published online: 26 January 2016

(C) Springer International Publishing AG 2016

\begin{abstract}
Penetrating abdominal vascular injuries result in significant mortality. In the pre-hospital phase of care, "scoop and run" is the optimal strategy while on-scene interventions are restricted only to basic airway maneuvers. Early in-hospital diagnosis and operative interventions are prerequisites for survival in vascular trauma. Hypotension and peritonitis following penetrating abdominal trauma mandate immediate access to the operating room with vascular surgery capabilities. Patients presenting in extremis should be managed with a resuscitative thoracotomy in the emergency department. The majority of abdominal vascular injuries surviving to the operating room are subjected to damage control intervention. In hemodynamically stable patients, diagnostic modality of choice is computed tomography angiography and endovascular interventions are evolving.
\end{abstract}

Keywords Abdominal vascular injury · Penetrating trauma . Management abdominal vessels

This article is part of the Topical Collection on Penetrating Injuries to Major Vessels

Peep Talving

peep.talving@ut.ee

1 Division of Acute Care Surgery, Department of Surgery, North Estonia Medical Center, J. Sütiste tee 19, 13419 Tallinn, Estonia

2 Department of Surgery, School of Medicine, University of Tartu, Tartu, Estonia

3 Department of Surgery, Tartu University Hospital, Tartu, Estonia

4 Division of Acute Care Surgery, Department of Surgery, Los Angeles County + University of Southern California Medical Center, Los Angeles, CA, USA

\section{Introduction}

Abdominal vascular injuries are associated with a significant on-scene mortality rate both in civilian and in combat settings. Historic study by DeBakey and colleagues reported that only $2 \%$ of all arterial injuries to the abdominal vessels to arrive to field hospitals in World War II [1]. Similarly, Rich and colleagues documented only $2.9 \%$ of combat-related abdominal vascular lesions per 1000 vascular injuries admitted to field hospitals during the Vietnam conflict [2]. Along the advancements of tactical combat casualty care, more severely injured military casualties survive to support hospitals with vascular injuries. Thus, Clouse and colleagues observed an incidence of $6.8 \%$ abdominal vascular lesions arriving to combat care hospitals in the Iraq conflict [3]. Likewise, in a study by Fox and co-authors, vascular torso injuries are admitted in increasing frequency in contemporary military conflicts [4].

In civilian settings, the recent multi-institutional investigation by DuBose and colleagues reported $7.8 \%$ vascular injuries to the intra-abdominal vessels admitted to 13 US trauma facilities $[5 \bullet \bullet$. The increasing incidence of vascular injuries arriving to medical care in the USA can be associated with truncated on-scene times, evolving prehospital management, and hemostatic resuscitation [6].

The overall in-hospital mortality of patients with abdominal vascular trauma ranges between 45 and $54 \%$ [7, 8]. Factors related to detrimental outcomes are the mechanism of injury, number of vessels injured, higher injury severity score, lower systolic blood pressure (SBP) on arrival, hypothermia, advanced age, and increased requirement of blood component transfusion $[7,8]$.

\section{General Principles of Management}

In abdominal vascular injury, early diagnosis and interventions are prerequisites for patient survival. In the 
prehospital phase of care, the best strategy is to "scoop and run." On-scene interventions should be restricted only to basic airway maneuvers. Insertion of an intravenous (IV) line is best attempted during transportation with similar success rates as compared to on-scene venous access [9]. Shorter prehospital times have resulted in more patients with abdominal vascular injuries reaching the hospital in extremis, thus increasing the overall in-hospital mortality following abdominal vascular trauma [10•].

Damage control resuscitation (DCR) throughout the preoperative phase of care is the emerging strategy for hypotensive patients sustaining penetrating trauma to the torso [9]. Nevertheless, the recent Cochrane analysis found no evidence for or against the use of early or smaller-volume IV fluid replacement in uncontrolled hemorrhage [11•]. However, the landmark study by Bickell and colleagues showed improved survival in delaying resuscitation in torso vascular injuries until arrival to the operating room [12]. Similarly, Cotton and co-authors noted survival benefit in patients requiring emergent trauma laparotomy subjected to DCR [13].

The optimal primary management follows Advanced Trauma Life Support (ATLS) principles. After definitive airway is secured and an adequate tissue perfusion is restored, mild hypotension is preferred until definitive treatment. IV access is accomplished via two large-bore peripheral IVs (16 gauge or larger) preferably in the upper extremities. Many trauma centers utilize large-bore double-lumen IV sheaths inserted into internal jugular or subclavian vein for access. It is of paramount importance to obtain vascular access that feeds into the superior vena cava (SVC) tributaries because abdominal vascular injury may include the inferior vena cava (IVC) that can preclude adequate vascular containment of infused volumes. Balanced volume replacement is provided early with blood products. Tranexamic acid should be given as early as possible in bleeding patients $[14,15 \cdot]$. Per the CRASH-2 study, in patients that receive tranexamic acid within the initial $3 \mathrm{~h}$ after the injury, risk of death due to bleeding was significantly lower; however, treatment later than $3 \mathrm{~h}$ may not be effective and may even be harmful [14]. The blood bank is notified immediately of the massive blood component requirement for 1:1:1 transfusion protocol [16•]. It has been noted, however, that fresh frozen plasma (FFP) administration in massive transfusion setting should be delayed until the first 6 units of red cell units are administered. In a study by Inaba et al., the authors noted that patients who did not require massive transfusion protocol (MTP) but received FFP too early suffered significantly more complications [17].

In parallel with resuscitation, Focused Assessment with Sonography for Trauma (FAST) is the instant diagnostic modality for both stable and unstable patients in the emergency department (ED) to rule in or out cardiac tamponade and hemoperitoneum. Abdominal X-ray can be used for hemodynamically stable patients with gunshot wound to the abdomen to delineate missile trajectory by placing markers (cardiac electrodes or paper clips) on entrance and exit wounds. Missile tract in proximity to any major vessel may provide information about the location of potential vascular injury. Computed tomographic angiography (CTA) is the diagnostic modality of choice only for hemodynamically stable patients with suspected abdominal vascular injury [18] (Fig. 1).

Hypotension following penetrating abdominal injury mandates immediate access to the operating room (OR) and an exploratory laparotomy incision from xiphoid to symphysis as the gold standard. When an immediate laparotomy is mandatory, a trauma OR with experienced staff should be readily available. Basic laparotomy and vascular trays should be available, and a table-mounted self-retaining retractor system provides optimal exposure. In sophisticated trauma facilities, autologous blood recovery and thoracic autotransfusion systems are frequently utilized.

Resuscitative thoracotomy and aortic cross-clamping with open cardiopulmonary resuscitation should be performed in the ED if the patient presents in extremis or in pulseless electrical activity; however, the survival rates of these patients are minimal [7] (Fig. 2). Resuscitative endovascular balloon occlusion of the aorta (REBOA) is a new emerging method for patients in end-stage hemorrhagic shock. Brenner et al. analyzed six cases where REBOA was performed and no hemorrhage-related mortalities or REBOA-associated complications were found [19•]. In a recent article by Biffl et al., an algorithm was proposed for the management of exsanguinating torso hemorrhage. These authors suggest a resuscitative thoracotomy for patients with imminent cardiac arrest and for patients with SBP $<60 \mathrm{mmHg}$. Patients with SBP 60 to $80 \mathrm{mmHg}$ should be subjected to an emergent surgical intervention. However, if an OR is not immediately available, a REBOA could be considered [20••].

Resuscitative laparotomy in the ED is controversial, and results are very poor. In a study by Mattox and colleagues, only $22 \%$ of patients subjected to resuscitative ED laparotomy reached the OR and mortality was $100 \%$ [21].

In patients who survive to the OR, the strategy of care is stratified dependent on the hemodynamic status and physiologic compromise. The majority of patients with abdominal vascular injury are subjected to damage control intervention to arrest the hemorrhage and to control contamination. Damage control options for a vascular injury are ligation or temporary intra-vascular shunting (TIVS) (Fig. 3). TIVS is utilized in end-organ vascular injury when ligation mandates end organ or extremity removal. There are commercial shunts available, or alternatively, a piece of plastic tube cut from a high-flow IV line, nasogastric tube, or a chest tube can be used. In the study from Groote Schuur Hospital, 22 patients had a TIVS inserted as a damage control procedure with overall mortality at 


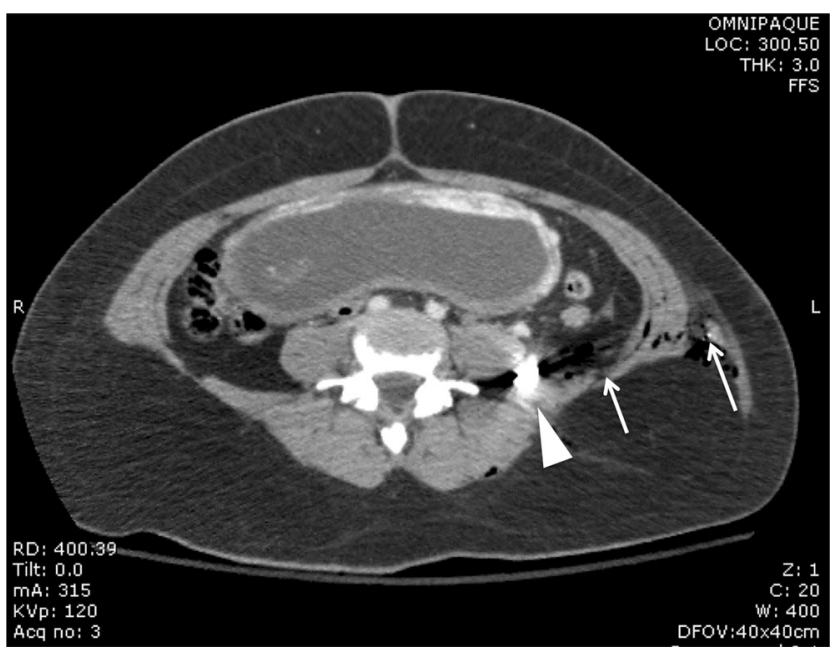

Fig. 1 Illustration depicts a missile tract (arrows) with bullet lodged in the retroperitoneal space (arrowhead) on a pregnant female

$22.7 \%$ [22]. In a study by Ball and Feliciano, TIVS and ligation for common and external iliac arterial injuries were compared observing significantly lower fasciotomy and amputation rates when TIVS was utilized [23].

The most commonly injured arteries in the abdomen are iliac vessels, followed by renal arteries and abdominal aorta; however, penetrating injuries accounted only $36.5 \%$ of the injuries per the PROOVIT registry [5••]. Asensio and colleagues observed that aortic injuries were most commonly followed by iliac arteries, superior mesenteric artery (SMA), renal arteries, splenic artery, celiac trunk, hepatic artery, and inferior mesenteric artery with penetrating mechanism contributing to $88 \%$ of injuries [7]. The most frequently major abdominal veins injured in descending order are IVC, iliac veins, renal veins, superior mesenteric vein (SMV), portal vein, hepatic veins, retrohepatic vena cava, splenic vein, and inferior mesenteric vein (IMV) [7].

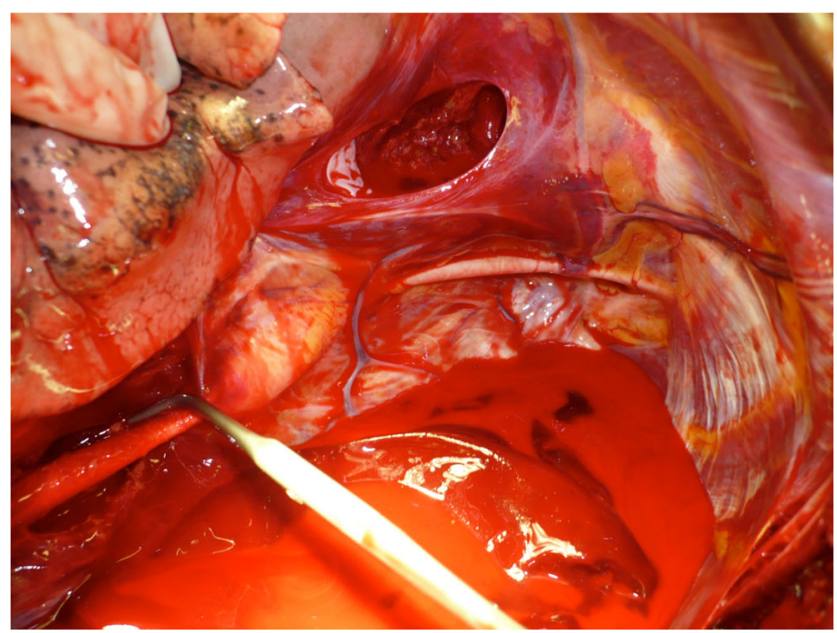

Fig. 2 Image depicting resuscitative emergency room thoracotomy and aortic cross-clamping in an arresting patient sustaining gunshot injury to the abdomen

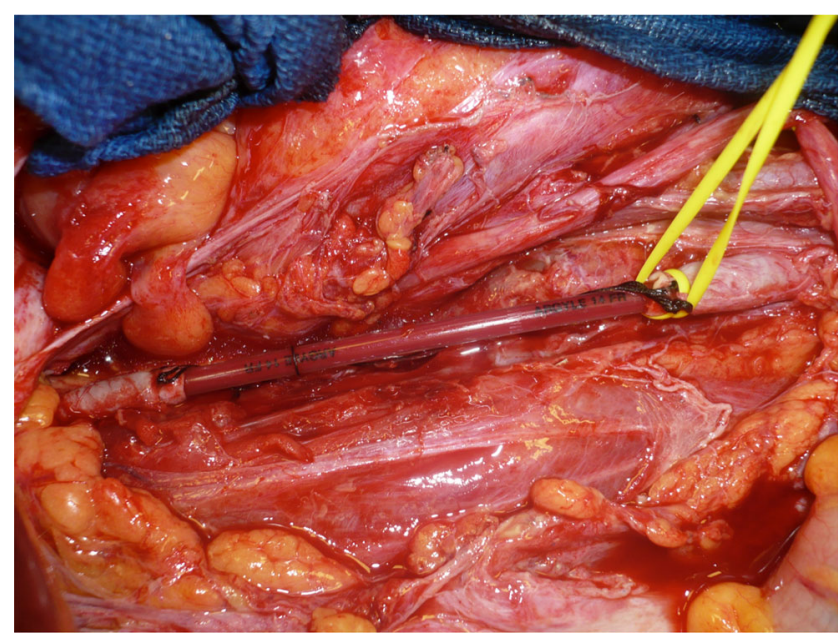

Fig. 3 Temporary intra-vascular shunt in the right external iliac artery in a patient sustaining a gunshot wound to the abdomen

Bleeding from intra-abdominal vessels can be either intraperitoneal, contained in the retroperitoneal space, or a combination of above. Retroperitoneal bleeding accounts for more than $90 \%$ of cases [7]. Distal SMA and branches of celiac trunk are intra-peritoneal with remaining major arteries being located in the retroperitoneal compartment. The retroperitoneal space is divided into functional zones that will help to diagnose the injury and plan an exploration during operation:

Zone I, supramesocolic region: suprarenal aorta, celiac axis, proximal superior mesenteric artery (SMA), superior mesenteric vein (SMV), renal artery

Zone I, inframesocolic region: infrarenal aorta, inferior mesenteric artery (IMA), and infrahepatic inferior vena cava (IVC)

Zone II: renal artery and vein

Zone III: common, internal, and external iliac arteries and veins

Zone IV: retrohepatic vena cava, portal vein, and hepatic artery

\section{Supramesocolic Zone I}

A hematoma or hemorrhage in the supramesocolic zone I is due to an injury of the abdominal aorta, celiac axis, SMA, or SMV. Injuries to the suprarenal aorta are associated with a mortality rate over $90 \%$ [8]. Nevertheless, if the abdominal aortic injury is contained in the retroperitoneal space, the patient may survive to the hospital. The best approach for the suprarenal aorta is a left medial visceral rotation popularized by DeBakey and Mattox [24]. The left colon is mobilized at the line of Toldt, the splenic flexure is taken down, and the spleen along with pancreatic tail is rotated to the midline 
exposing the abdominal aorta with its branches in the retroperitoneal space. Proximal control of the aorta at the hiatus of the diaphragm can be achieved by pressure with a sponge stick, a compression device, or by digital control. Aortic cross-clamping to the lowest segment of the thoracic aorta is the next step after dividing some left crural muscle fibers.

Another option for aortic proximal control is supraceliac cross-clamping achieved through the lesser sac. The left triangular ligament of the liver is taken down, the esophagus that contains a nasogastric tube is identified, and the structure just to the patient right of the esophagus is the aorta. The left crus of the diaphragm is divided at 2 o'clock where it is avascular so the low thoracic aorta can be exposed for cross-clamping. The backflow is controlled by distal cross-clamping proximal to the bifurcation of the aorta (Fig. 4). Time of inflow control should be noted and kept as short as possible. Prolonged aortic clamping is associated with an induced fibrinolysis and more profound reperfusion injury $[25,26]$. After obtaining proximal and distal control, the first option for repair is a lateral aortorrhaphy with 3-0 or 4-0 prolene suture. Often, if the damaged part is large or resection is needed, primary suture may cause a significant narrowing of the aorta and a polytetrafluoroethylene (PTFE) patch or an end-to-end anastomosis with a Dacron graft is the remaining choice. Omental covering of the anastomosis should be considered. Primary end-to-end anastomosis is almost impossible due to limited mobility of aorta. Ligation of the aorta is not an option. Some surgeons have attempted temporary aortic shunting using a chest tube with limited success (Fig. 5).

Injuries of the celiac axis are rare. In a study from Los Angeles County General Hospital, 13 patients reached the hospital with celiac axis injury during 132-month period and $92 \%$ of those patients had sustained a penetrating trauma. The survival rate was $38 \%, 80 \%$ of the surviving patients were treated with ligation [27]. The ligation of celiac axis is well tolerated due to extensive collateral circulation; however, ischemia of the gallbladder may occur requiring cholecystectomy when hemodynamic stability is ensured [28]. For the limited injuries, primary repair is recommended and ligation should be reserved for more destructive injuries. Complex repairs with an end-to-end anastomosis, autologous interposition graft, or synthetic graft repairs are not recommended as the morbidity with celiac axis ligation is low [27].

The SMA requires more extensive approach to prevent detrimental bowel ischemia. Fullen described four anatomical zones of SMA to unify the management [29]. Zone I is beneath the pancreas, zone is II between the pancreaticoduodenal complex and middle colic branches of the artery, zone III is beyond the middle colic branches, and zone IV is at the level of enteric branches. The more proximal the location of injury, the higher the mortality, ranging from $76.5 \%$ for the zone I and $23.1 \%$ for the zone IV injuries, respectively [30]. Exposure of zone I and zone II injuries may

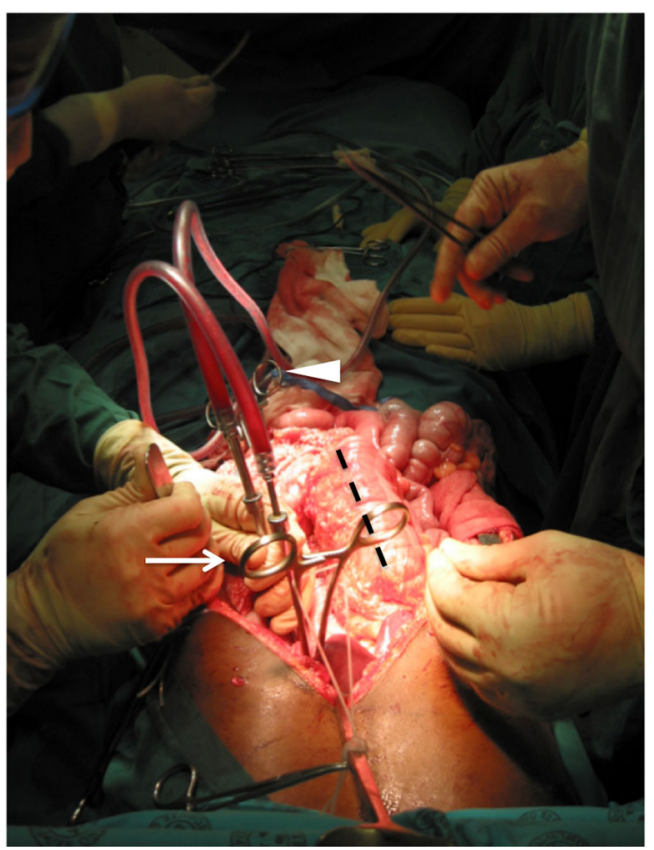

Fig. 4 Proximal and distal control of abdominal aortic gunshot injury at a damage control laparotomy. Medial visceral rotation of the left colon is demonstrated with a dashed line. Arrow and arrowhead indicate the proximal clamp and the distal clamp, respectively

require transection of the pancreatic neck after the proximal control of the aorta is achieved by supraceliac aortic crossclamping. When accessed through the left medial visceral rotation, the repair is feasible without dividing the pancreas.

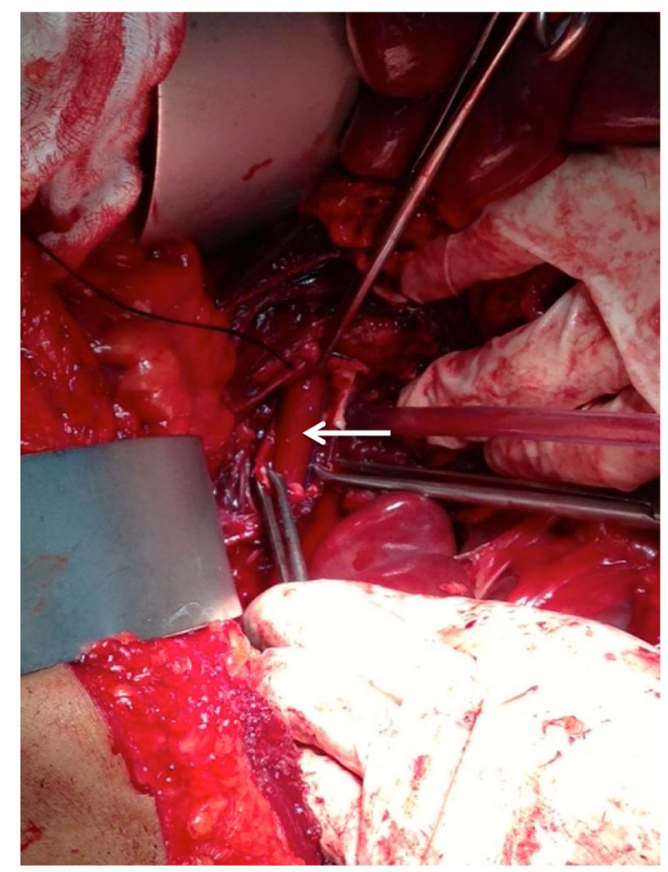

Fig. 5 Chest tube used as a temporary intra-vascular shunt following gunshot injury to the abdominal aorta. Arrow points to the shunt 
Zone III and zone IV injuries can be achieved by reflecting the transverse colon in a cranial direction accessing the SMA at the mesenteric root distal to the transverse mesocolon. SMA injuries need to be repaired primarily, reimplanted to the aorta, or managed with an interposition graft. In the damage control setting, temporary shunting is the best option.

Half of the patients with penetrating SMV injury have a small bowel or large bowel injury concomitantly. In $42 \%$ of the cases, SMV injury is present along with SMA lesion [31]. More recent studies have reported mortality rates ranging between 44 and $57 \%$ [7, 31]. The SMV is located to the right of the SMA and can be accessed by dividing the ligament of Treitz between the fourth portion of duodenum and the base of the transverse mesocolon. If the exposure is still not sufficient, pancreatic transection provides access to the vessel. The options after identifying the injury are suture repair, autologous vein graft, ligation, or shunting, depending on the condition of the patient and extent of the injury (Fig. 6). The splenic vein turndown procedure has been reported as an alternative method for large defects [32]. For the unstable patients, ligation is an acceptable option; however, massive postoperative fluid therapy is needed and an open abdomen approach should be considered due to massive small bowel edema and subsequent abdominal compartment syndrome [33].

\section{Inframesocolic Zone I}

Most commonly injured vascular structures in the inframesocolic zone I are the infrarenal aorta and IVC. About half of the aortic injuries are infrarenal and are associated with an almost $70 \%$ mortality rate [8]. The exposure of an inframesocolic hematoma is similar to maneuvers utilized during elective abdominal aortic aneurysm repair. The transverse mesocolon is retracted in a cranial direction, the small bowel is eviscerated to the right side, and the midline retroperitoneum is opened and dissected until the left renal vein is exposed. The proximal control of the injury is obtained by placing the aortic clamp right below the left renal vein. The distal control is accomplished by clamping the aorta just proximal to the bifurcation. In less extensive injuries, a side-biting Satinsky clamp provides hemorrhage control and suture repair (Fig. 7). If necessary, the IMA can be ligated with impunity. The repair of inframesocolic aortic injury is similar to supramesocolic injury with possible lateral arteriorraphy, PTFE patching, or with Dacron interposition graft in those very limited cases surviving to hospital admission.

Injury to the IVC usually presents with a hematoma to the right of midline. Access to the infrahepatic IVC is best accomplished by a right medial visceral rotation popularized by Cattell and Braasch performed along with the Kocher maneuver. Prior to the exposure, sponges-on-sticks are prepared for proximal and distal compression of the IVC (Fig. 8). Side-

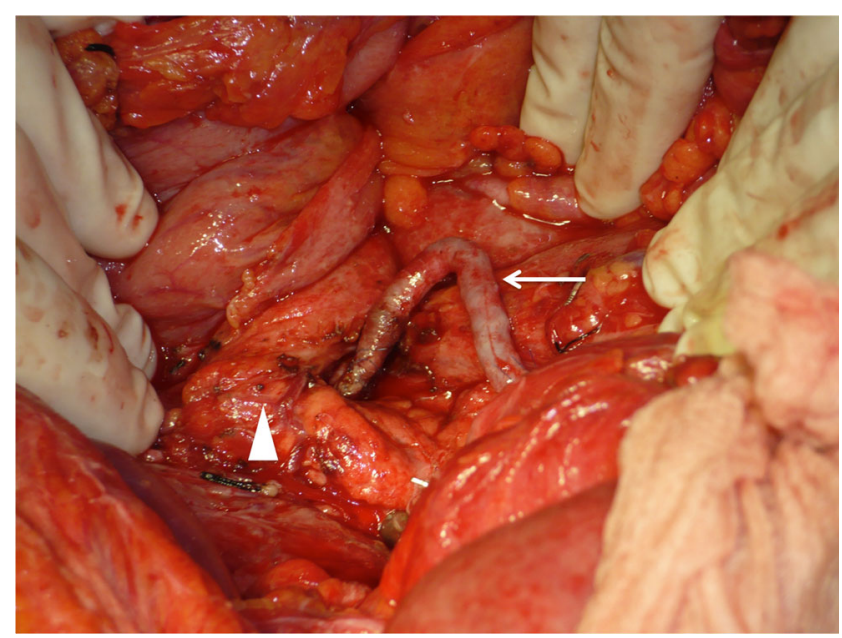

Fig. 6 Autologous vein graft utilized in a destructive superior mesenteric vein injury. Arrow indicates the autologous graft, and arrowhead points to the neck of the pancreas

biting Satinsky clamp is very useful to control the hemorrhage and to allow suture line placement along the clamp. First-line therapy is lateral repair. If the primary suture line causes stenosis of $50 \%$ or more, a PTFE patch should be used. Ligation for complex infrarenal IVC injuries is generally accepted when patient is physiologically compromised while ligation of suprarenal IVC injuries is associated with renal failure and has very few long-term survivors [34-36]. In a study by Sullivan et al., overall mortality of patients undergoing infrarenal IVC ligation was significantly higher than those undergoing primary repair (59 vs. $21 \%$ ) and $77 \%$ of ligation group patients needed below-knee fasciotomy [34]. However, in a study by Navsaria et al. from South Africa, there was no significant difference in mortality between ligation vs. repair group and none of the patients needed fasciotomy [37]. Postligation elastic compression stockings and leg elevation are

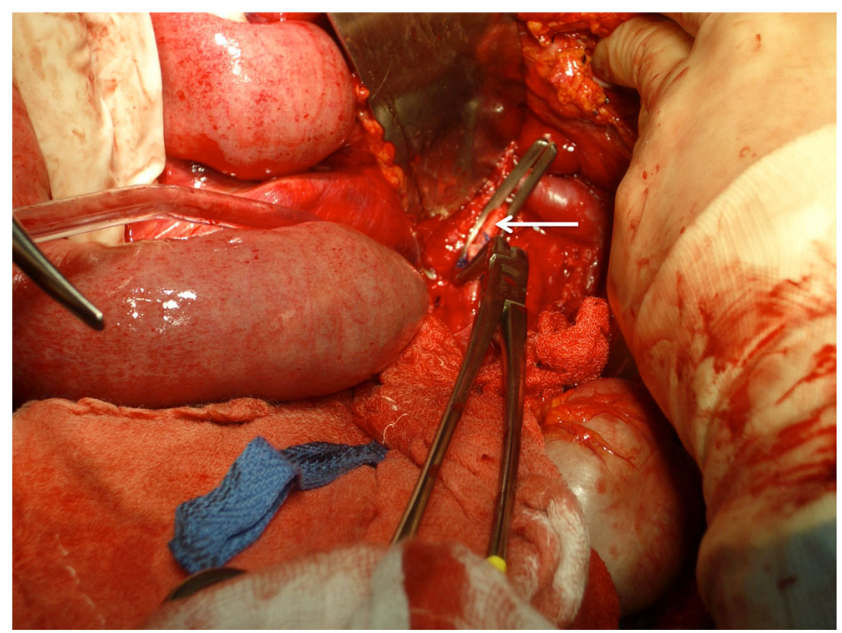

Fig. 7 Aortic stab wound (arrow) controlled and with a side-biting Satinsky clamp 
always necessary and should be commenced on the operating table. Complex reconstruction procedures with prosthetic graft or saphenous vein have been described, but the numbers are too small to make any conclusive suggestions $[38,39]$.

Associated intra-abdominal injuries are often present, with small bowel and liver injuries being the most frequent [37]. In the pediatric population, IVC injuries are reported as the most common venous injuries [40•].

\section{Zone II}

There are two approaches for renovascular injuries - medial or lateral approach — with the latter being faster. For the lateral approach, the injured kidney is approached by entering the retroperitoneum laterally at the line of Toldt and mobilizing the colon. Gerota's fascia is opened with sharp and blunt dissection, and the kidney is mobilized to midline followed by digital control or clamping of the hilum.

For the compromised patient with two kidneys, nephrectomy is best implemented if a renal arterial injury is found and the contralateral kidney is confirmed by palpation of the contralateral retroperitoneal compartment. If the patient is stable, renal arterial injury can be managed with a lateral arteriorraphy or with end-to-end anastomosis. Graft repair is rarely described in the trauma setting, and every minute of warm ischemia associated with hemodynamic compromise is detrimental to the renal unit. Each additional minute of warm ischemia time is found to increase the risk of renal failure in non-trauma settings and therefore makes complex arterial repairs questionable [41]. Mortality due to renal artery injury is about $36.4 \%$ [7].

Renal vein injuries are best managed with primary lateral repair if possible. The left renal vein can be ligated safely between IVC and the left gonadal vein if the left gonadal

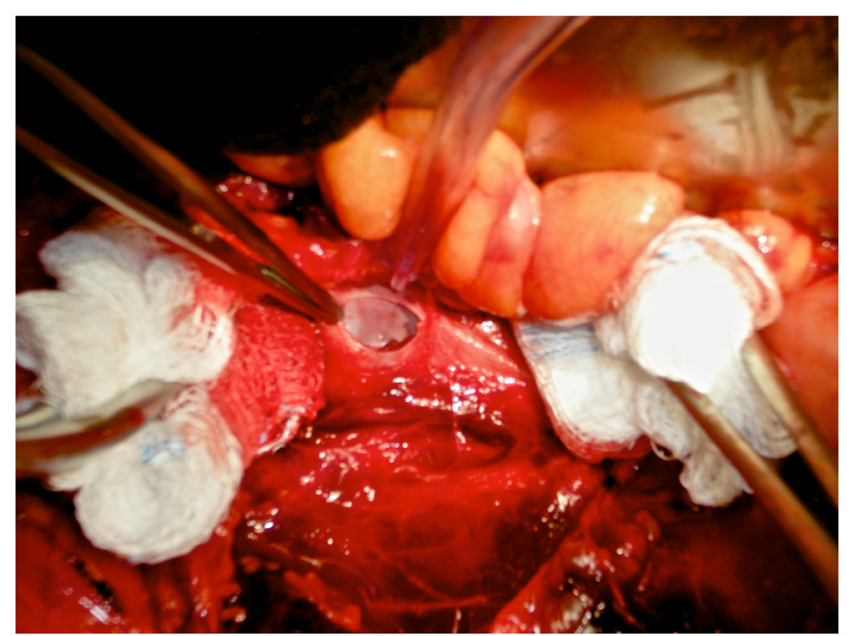

Fig. 8 Sponges on sticks utilized for proximal and distal control of a gunshot wound to the inferior vena cava and adrenal veins are intact, however, nephrectomy should be performed if a left renal arterial injury is also present. Ligation of the right renal vein mandates nephrectomy. Mortality after renal vein injury is reported to be $44 \%$ [7].

\section{Zone III}

Hematomas in the pelvic retroperitoneum, or zone III, are associated with common, external, or internal iliac vessel injuries (Fig. 9). Following penetrating trauma with zone III retroperitoneal injury, iliac vascular injury is present until proven otherwise. Iliac vessels are approached by eviscerating the small bowel to the right and dividing the retroperitoneum over the aortic bifurcation. Initial control should be obtained digitally or by sponge-stick with subsequent proximal and distal control by clamping. Ureter coursing over the iliac vessels must be identified and preserved. In difficult-to-control bleeding from the iliac vessels following transpelvic gunshot wounds, pelvic vascular isolation or "walking the clamps" is an option. This technique requires four clamps placed on proximal common iliac vessels and to the distal external iliac vessels. In achieving initial hemostasis, the distal clamps are walked proximally until the location of the injury is identified and controlled. The remaining clamps are subsequently removed. The common and external iliac artery injuries are repaired with lateral repair or with a PTFE interposition graft. In the damage control setting, temporary shunting is a very viable option. Ligation of common and external iliac artery is associated with $47 \%$ of amputation rate [23]. Internal iliac arteries can be ligated safely, even bilaterally.

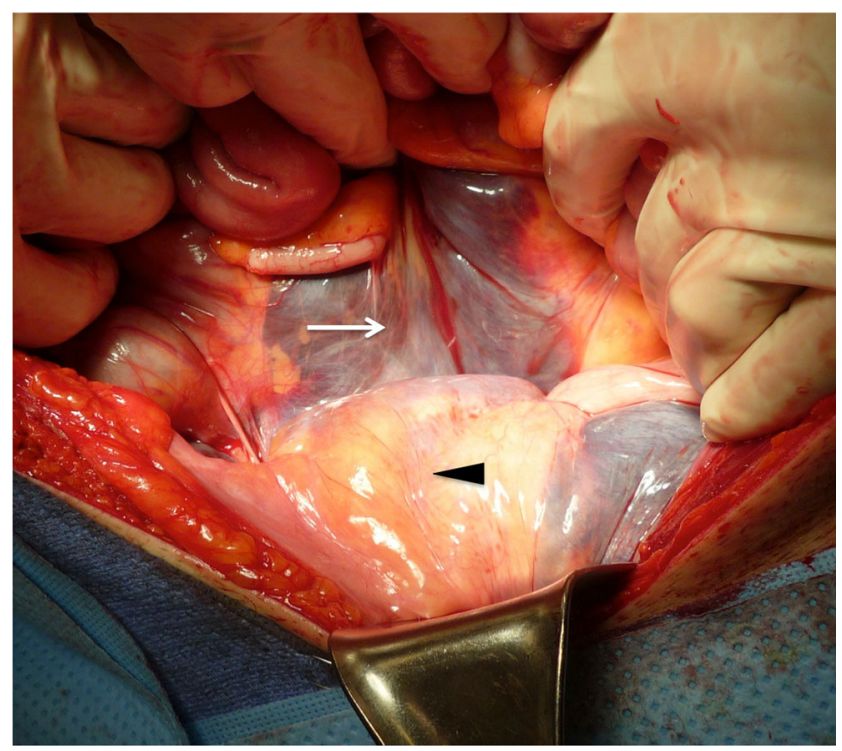

Fig. 9 Zone III retroperitoneal hematoma consistent with transpelvic gunshot wound and external iliac artery injury. Arrow points to the hematoma and arrowhead to the bladder 
Injuries to the iliac veins can be managed by lateral venorrhaphy or ligation.

\section{Zone IV}

Retrohepatic IVC and hepatic vein insults are some of the most devastating abdominal vascular injuries with mortality rate beyond $90 \%$ [7]. Exploration of contained retrohepatic hematoma should be avoided if at all possible; however, decompressed hematomas with massive bleeding require exploration and intervention. The first best option is perihepatic packing. If packing does not work, hepatic isolation with subdiaphragmatic aortic cross-clamping followed by clamping of the suprarenal and suprahepatic IVC in addition to a Pringle maneuver is the next possible maneuver. The last option is atriocaval shunting with very few successful case reports in the literature [34, 42-44].

Hematoma in the portal triad is associated with injured hepatic artery or portal vein. Before entering the hematoma, the Pringle maneuver should be applied. In the damage control setting, the hepatic artery can be ligated; however, the portal vein has to be intact. Cholecystectomy should be performed if the hepatic artery is ligated.

Mortality in portal vein injury is about $70 \%$ [7]. Access to the portal vein is obtained by right medial visceral rotation followed by the Kocher maneuver. Stapled pancreatic division may be required if the injured part of the portal vein is located behind the pancreatic neck. Iatrogenic injuries to the hepatic artery or common bile duct should be avoided during the exposure. The first option for injured portal vein is lateral repair if at all feasible and hemodynamics are favorable. In an exsanguinating patient, early ligation may be preferable. The survival rate is somewhat higher if the decision to ligate is done very early. However, intact hepatic artery is required if ligation of portal vein is performed [45]. The abdomen should be left open to avoid abdominal compartment syndrome, and a massive resuscitation may be required due to splanchnic bed fluid sequestration following portal vein ligation.

\section{Conclusion}

Abdominal vessel injuries are becoming more common as the prehospital care has advanced. Management of these injuries mandates familiarity with the anatomy and quick repair by the surgeon. The vessels are mostly retroperitoneal and are divided into four zones. These zones assist in diagnosis of the vessel injured and the proposed repair of the injury. Suture repair, patch, or end-end anastomosis is the variety of options in the hemodynamically stable patient for the majority of vascular injuries. Ligation of the injured vessel may be necessary in the hemodynamically unstable patient; however, vascular shunts should be considered in those vessels that cannot be ligated with impunity.

\section{Compliance with Ethical Standards}

Conflict of Interest Drs. Peep Talving, Lydia Lam, and Sten Saar declare no conflicts of interest.

Human and Animal Rights and Informed Consent This article does not contain any studies with human or animal subjects performed by any authors.

\section{References}

Papers of particular interest, published recently, have been highlighted as:

- Of importance

•- Of major importance

1. Michael E, DeBakey FAS. Battle injuries of the arteries in World War II: an analysis of 2,471 cases. Ann Surg. 1946;123(4):534.

2. Rich NM, Baugh JH, Hughes CW. Acute arterial injuries in Vietnam: 1,000 cases. J Trauma Acute Care Surg. 1970;10(5):359.

3. Clouse WD, Rasmussen TE, Peck MA, Eliason JL, Cox MW, Bowser AN, et al. In-theater management of vascular injury: 2 years of the Balad Vascular Registry. J Am Coll Surg. 2007;204(4):625-32.

4. Fox CJ, Patel B, Clouse WD. Update on wartime vascular injury. Perspect Vasc Surg Endovasc Ther. 2011;23(1):13-25.

5.• DuBose JJ, Savage SA, Fabian TC, Menaker J, Scalea T, Holcomb JB. The American Association for the Surgery of Trauma PROspective Observational Vascular Injury Treatment (PROOVIT) registry: multicenter data on modern vascular injury diagnosis, management and outcomes. J Trauma Acute Care Surg. 2015;78(2):215-22. A recent study based on a large PROspective Vascular Injury Treatment (PROOVIT) registry invlolving 13 Level I and one Level II American College of Surgeons verified trauma centers.

6. Fox CJ, Bowman JN. Advances in resuscitation in the setting of vascular injury. Perspect Vasc Surg Endovasc Ther. 2011;23(2): $112-6$.

7. Asensio JA, Chahwan S, Hanpeter D, Demetriades D, Forno W, Gambaro E, et al. Operative management and outcome of 302 abdominal vascular injuries. Am J Surg. 2000;180(6):528-34.

8. Tyburski JG, Wilson RF, Dente C, Steffes C, Carlin AM. Factors affecting mortality rates in patients with abdominal vascular injuries. J Trauma Acute Care Surg. 2001;50(6):1020.

9. Smith RM, Conn AK. Prehospital care - scoop and run or stay and play? Injury. 2009;40:S23-6.

10. Ball CG, Williams BH, Tallah C, Salomone JP, Feliciano DV. The impact of shorter prehospital transport times on outcomes in patients with abdominal vascular injuries. J Trauma Manag Outcomes. 2013;7(1):11. This study compared prehospital transportation times of two periods of time (1991-1994 vs. 19952004) and showed that reduction in transport times resulted in an increase in the number of patients arriving with abdominal vascular injuries, the proportion presenting in physiologic extremis, and overall mortality. 
11. Kwan I, Bunn F, Chinnock P, Roberts I. Timing and volume of fluid administration for patients with bleeding. Cochrane Database Syst Rev. 2014;5(3):CD002245. A large study involving six randomised trials comparing different resuscitation strategies in trauma patients with hemorrhagic shock.

12. Bickell WH, Wall Jr MJ, Pepe PE, Martin RR, Ginger VF, Allen MK, et al. Immediate versus delayed fluid resuscitation for hypotensive patients with penetrating torso injuries. N Engl J Med. 1994;331(17):1105-9.

13. Cotton BA, Reddy N, Hatch QM, LeFebvre E, Wade CE, Kozar $\mathrm{RA}$, et al. Damage control resuscitation is associated with a reduction in resuscitation volumes and improvement in survival in 390 damage control laparotomy patients. Ann Surg. 2011;254(4):598605.

14. CRASH-2 collaborators, Roberts I, Shakur H, Afolabi A, Brohi K, Coats T. The importance of early treatment with tranexamic acid in bleeding trauma patients: an exploratory analysis of the CRASH-2 randomised controlled trial. Lancet. 2011;377(9771):1096-101.

15. Ker K, Roberts I, Shakur H, Coats TJ. Antifibrinolytic drugs for acute traumatic injury. Cochrane Database Syst Rev. 2015;9(5): CD004896. This study involved three trials evaluating effect of antifibrinolytic drugs following traumatic injury.

16. Holcomb JB, Tilley BC, Baraniuk S, Fox EE, Wade CE, Podbielski JM, et al. Transfusion of plasma, platelets, and red blood cells in a 1: $1: 1$ vs a $1: 1: 2$ ratio and mortality in patients with severe trauma. JAMA. 2015;313(5):471-82. This study compared a 1:1:1 and 1: 1:2 massive transfusion protocols during active resuscitation. More patients in the 1:1:1 group achieved hemostasis and fewer experienced death due to exsanguination by 24 hours.

17. Inaba $\mathrm{K}$, Branco BC, Rhee P, Blackbourne LH, Holcomb JB, Teixeira PG, et al. Impact of plasma transfusion in trauma patients who do not require massive transfusion. J Am Coll Surg. 2010;210(6):957-65.

18. Patterson BO, Holt PJ, Cleanthis M, Tai N, Carrell T, Loosemore TM, et al. Imaging vascular trauma. Br J Surg. 2011;99(4):494 505.

19. Brenner ML, Moore LJ, DuBose JJ, Tyson GH, McNutt MK, Albarado RP, et al. A clinical series of resuscitative endovascular balloon occlusion of the aorta for hemorrhage control and resuscitation. J Trauma Acute Care Surg. 2013;75(3):506-11. Descriptive case series about an implementation of REBOA in civilian trauma centers.

$20 . \bullet$ Biffl WL, Fox CJ, Moore EE. The role of REBOA in the control of exsanguinating torso hemorrhage. J Trauma Acute Care Surg. 2015;78(5):1054-8. Recently published article proposing an algorithm for the management of patients with exsanguinating torso hemorrhage.

21. Mattox KL, Allen MK, Feliciano DV. Laparotomy in the emergency department. JACEP. 1979;8(5):180-3.

22. Oliver JC, Gill H, Nicol AJ, Edu S, Navsaria PH. Temporary vascular shunting in vascular trauma: a 10-year review from a civilian trauma centre. S Afr J Surg. 2013;51(1):6-10.

23. Ball CG, Feliciano DV. Damage control techniques for common and external iliac artery injuries: have temporary intravascular shunts replaced the need for ligation? J Trauma. 2010;68(5): $1117-20$.

24. Rasmussen TE, Tai NRM. Rich's vascular trauma. Elsevier; 2015.

25. Illig KA, Green RM, Ouriel K, Riggs PN, Bartos S, Whorf R, et al. Primary fibrinolysis during supraceliac aortic clamping. J Vasc Surg. 1997;25(2):244-54.
26. Haithcock BE, Shepard AD, Raman SBK, Conrad MF, Pandurangi $\mathrm{K}$, Fanous NH. Activation of fibrinolytic pathways is associated with duration of supraceliac aortic cross-clamping. J Vasc Surg. 2004;40(2):325-33.

27. Asensio JA, Petrone P, Kimbrell B, Kuncir E. Lessons learned in the management of thirteen celiac axis injuries. South Med J. 2005;98(4):462-6.

28. Kavic SM, Atweh N, Ivy ME, Possenti PP, Dudrick JS. Celiac axis ligation after gunshot wound to the abdomen: case report and literature review. J Trauma Acute Care Surg. 2001;50(4):738.

29. Fullen WD, Hunt J, Altemeier WA. The clinical spectrum of penetrating injury to the superior mesenteric arterial circulation. J Trauma Acute Care Surg. 1972;12(8):656.

30. Asensio JA, Britt LD, Borzotta A, Peitzman A, Miller FB, Mackersie RC, et al. Multiinstitutional experience with the management of superior mesenteric artery injuries. J Am Coll Surg. 2001;193(4):354-65.

31. Asensio JA, Petrone P, Garcia-Nunez L. Superior mesenteric venous injuries: to ligate or to repair remains the question. J Trauma. 2007;62(3):668-75.

32. Phillips BT, Pasklinsky G, Watkins KT, Vosswinkel JA, Tassiopoulos AK. Splenic vein turndown repair in superior mesenteric vein trauma: a reasonable alternative. Vasc Endovasc Surg. 2011;45(2):191-4.

33. Goaley TJ, Dente CJ, Feliciano DV. Torso vascular trauma at an urban level I trauma center. Perspect Vasc Surg Endovasc Ther. 2006;18(2):102-12.

34. Sullivan PS, Dente CJ, Patel S, Carmichael M, Srinivasan JK, Wyrzykowski AD, et al. Outcome of ligation of the inferior vena cava in the modern era. Am J Surg. 2010;199(4):500-6.

35. Ivy ME, Possenti P, Atweh N, Sawyer M, Bryant G, Caushaj P. Ligation of the suprarenal vena cava after a gunshot wound. J Trauma. 1998;45(3):630-2.

36. Votanopoulos KI, Welsh FJ, Mattox KL. Suprarenal inferior vena cava ligation: a rare survivor. J Trauma Acute Care Surg. 2009;67(6):E179-80.

37. Navsaria PH, De Bruyn P, Nicol AJ. Penetrating abdominal vena cava injuries. Eur J Vasc Endovasc Surg. 2005;30(5):499-503.

38. Oldhafer KJ, Frerker M, Winkler M, Schmidt U. Complex inferior vena cava and renal vein reconstruction after abdominal gunshot injury. J Trauma. 1999;46(4):721-3.

39. Nigro J, Velmahos GC. Delayed reconstruction of the inferior vena cava with prosthetic graft due to postligation edema. Contemp Surg. 1999;54:25-7.

40. Rowland SP, Dharmarajah B, Moore HM, Dharmarajah K, Davies AH. Venous injuries in pediatric trauma. J Trauma Acute Care Surg. 2014;77(2):356-63. Systematic review invlolving thirteen articles about venous injuries in a pediatric population.

41. Thompson RH, Lane BR, Lohse CM, Leibovich BC, Fergany A, Frank I. Every minute counts when the renal hilum is clamped during partial nephrectomy. Eur Urol. 2010;58(3):340-5.

42. Clark JJ, Steinemann S, Lau JM. Use of an atriocaval shunt in a trauma patient: first reported case in Hawai 'i. Hawaii Med J. 2010;69(2):47-8.

43. Soto S, Oettinger R. Atriocaval shunt. Report of two cases. Rev Med Chil. 2005;133(3):327-30.

44. Burch JM, Feliciano DV, Mattox KL. The atriocaval shunt. Facts Fict Ann Surg. 1988;207(5):555.

45. Talving P, Inaba K. Major abdominal veins. In: Velmahos GC, Degiannis E, Doll D, editors. Penetrating trauma, Springer. 2012. 